\title{
Effect of grain shape on the jamming of two-dimensional silos
}

\author{
Ezequiel Goldberg ${ }^{1, \star}$, C. Manuel Carlevaro ${ }^{1,2, \star \star}$, and Luis A. Pugnaloni ${ }^{3, \star \star \star}$ \\ ${ }^{1}$ Universidad Tecnológica Nacional - FRBA, UDB Física, Mozart 2300, C1407IVT Buenos Aires, Argentina. \\ ${ }^{2}$ Instituto de Física de Líquidos y Sistemas Biológicos (CONICET La Plata, UNLP), Calle 59 Nro 789, 1900 La Plata, Argentina. \\ ${ }^{3}$ Dpto. Ingeniería Mecánica, Facultad Regional La Plata, Universidad Tecnológica Nacional, CONICET, Av. 60 Esq. 124,1900 \\ La Plata, Argentina.
}

\begin{abstract}
We present discrete element method simulations of the discharge of silos in two dimensions. We study the effect of the grain shape on the clogging of small apertures, considering regular polygons and disks of equal mass. In particular, we analyze the avalanche size distribution and the jamming probability for disks, triangles, squares, pentagons, hexagons and heptagons as a function of the aperture size. We show that the jamming probability presents a non-linear response as a function of the number of vertexes of the polygons.
\end{abstract}

\section{Introduction}

The clogging (or jamming) of the flow of grains through small apertures is usually an undesired phenomenon in different industrial processes. The search for a prediction of a minimum suitable opening size that warrants that clogging will not occur in the flow has been driven by practical reasons. It is generally accepted that, for spherical grains, a circular opening larger than five particle diameters will rarely clog. Whether this is a sharp transition is still under debate $[1,2]$. The friction coefficient of the grain-grain interaction seems to play little role for spheres [3]. However, one should expect that the shape of the grains is a key factor for the ability of grains to clog. Indeed, Zuriguel et al. [3] have shown that rice (elongated grains) and pasta pellets (short cylindrical grains) do present a larger tendency to jamming. Despite these initial investigations, there has not been systematic studies on the effect of particle shape on clogging.

In this work, we present results of two-dimensional simulations of grains with shape of regular polygons (triangles, squares, pentagons, hexagons and heptagons) in addition to circular grains. We focus on the clogging events during the discharge of the grains trough small openings in the base of a flat bottom silo. To compare results from different shapes we use as a criterion that the grains must have the same mass. We will show that the intuitive prediction that polygonal grains are more prone to clogging than disks is indeed confirmed. However, triangular particles show a marked reduction in clogging if compared to squares, pentagons and hexagons.

\footnotetext{
^e-mail: ezequiel.goldberg@outlook.com.ar

$\star \star$ e-mail: manuel@iflysib.unlp.edu.ar

$\star \star \star$ e-mail: luis.pugnaloni@ frlp.utn.edu.ar
}

\section{Simulation}

We simulate a 2D silo by means of a discrete element method (DEM) using the Box2D library [4]. This package uses a constraint solver. At each time step of the dynamics, a series of iterations (typically 20) are used to resolve penetrations between bodies through a Lagrange multiplier scheme [5]. After detecting overlaps, the inelastic collision at each contact is solved and new linear and angular velocities are assigned. The equations of motion are integrated through a symplectic Euler algorithm. The time step $\delta t$ used to integrate the equations of motion is 0.025 $d / g$; with $d$ the diameter of our circular grains and $g$ the acceleration of gravity. Solid friction is also handled by means of a Lagrange multiplier scheme that implements the Coulomb criterion. Box2D has been previously used to simulate continuous silo discharges [6], and tapping of granular systems [7].

The silo consist of a box $20 d$ wide and $180 d$ high. Gravity acts in the negative vertical direction. 2000 grains are used in each discharge, and these are not reinjected after they leave the silo. After grains initially placed at ran-



(a)



(b)

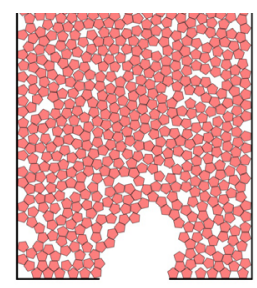

(c)
Figure 1. Snapshots of the simulations after a clog is detected: (a) triangles, (b) squares and (c) pentagons. Only the region close to the aperture is shown. 


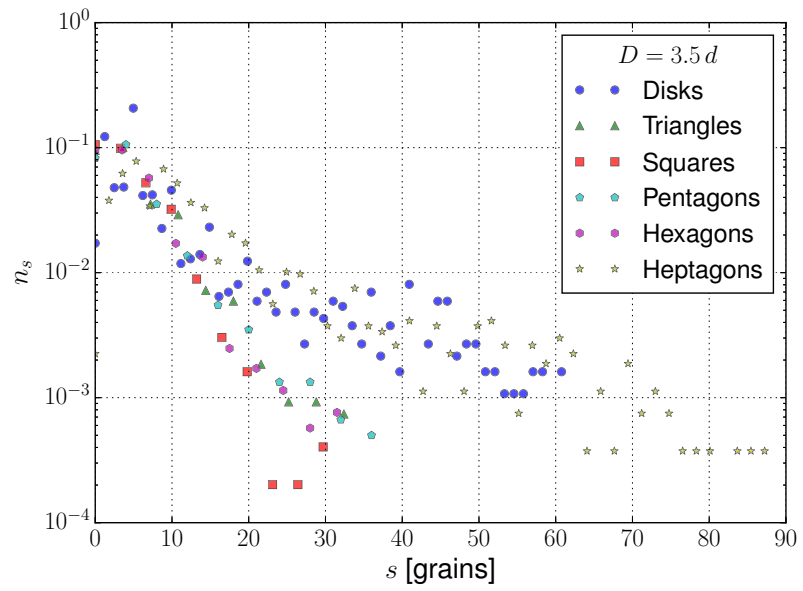

Figure 2. Normalized distribution of avalanche sizes $n_{s}(D)$ for an aperture of width $D=3.5 d$ for different grain shapes (see legend).

dom without overlaps settle, an aperture opened at the center of the flat base of the silo allows the grains to discharge. For small apertures, clogging is observed. We consider a clog is permanent if more than 5 seconds have passed without any grain falling through the aperture. For each clogging we record the number of particles that passed through the aperture. We do not resume the discharge by perturbing the clogged system, instead, we carry out a new simulation by filling a new silo using different random initial positions for the grains. We record, for each aperture size, and for each grains shape, 1500 clogs. For large aperture sizes the entire system discharges without clogging. In these cases we record that an avalanche larger that 2000 grains has occurred.

Grains of different shapes corresponding to regular polygons (triangles, squares, pentagons, hexagons and heptagons) are used in the simulations apart from the circular grains. All grains have the same material density and the same area, hence the same weight. Each simulation contains only one type of grain and these are monosized. Figure 1 shows some snapshots of systems after a clog. The restitution coefficient is set in all cases to $\epsilon=0.05$ and the friction coefficient $\mu$ is set to 0.5 . The significantly low restitution coefficient allows for a rapid dissipation of the kinetic energy during the initial filling of the silo. The material density $\rho$ of the grains is set to $0.01 \mathrm{Kg} / \mathrm{m}^{2}$

\section{Results}

We consider the distribution of avalanche sizes $n_{s}(D)$ which is the number of avalanches of $s$ grains. Figure 2 shows the normalized $n_{s}$ for an aperture $D=3.5 d$, for each grain shape. As we can see, most polygonal shapes show a similar size distribution of the avalanches. There is however a marked difference with circular grains, which tend to deliver much larger avalanches for a given orifice. Interestingly, heptagons seem to have avalanches similar to disks. This may be due to the polygon having enough edges to behave close to a disk. We have not explored
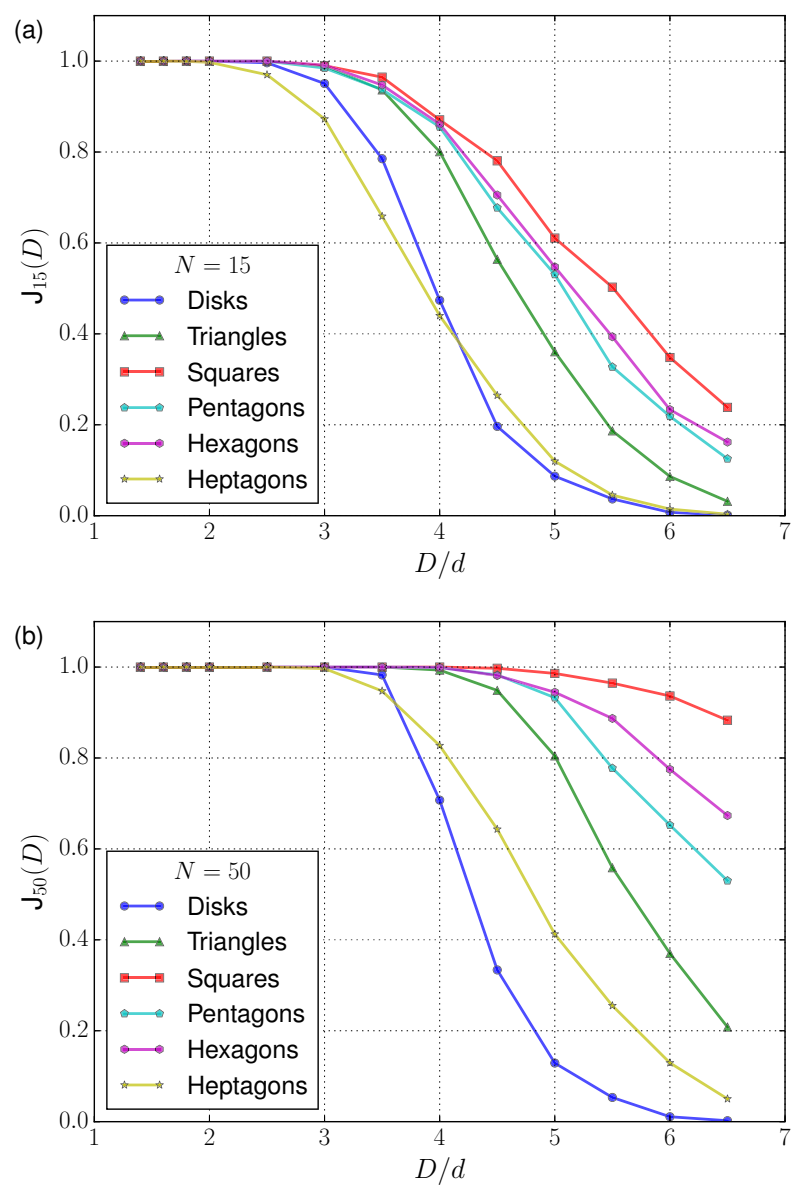

Figure 3. Jamming probability for the different grain shapes for (a) $N=15$, and (b) $N=50$.

polygons with larger number of vertexes to confirm if this is the case. The distributions have an exponential tail, consistent with all previous results on disks and spheres (see for example Refs. [1, 3, 8]

We also calculate the jamming probability $J_{N}(D)$ defined as [3]

$$
J_{N}(D)=\frac{\sum_{s=N}^{\infty} s n_{s}(D)}{\sum_{s=1}^{\infty} n_{s}(D)} .
$$

$J_{N}(D)$ corresponds to the probability that an avalanche smaller that $N$ grains discharges thought an aperture of size $D$ before the system clogs. Figure 3 shows $J_{15}(D)$ and $J_{50}(D)$ for different grain shapes. As observed in previous studies, $J_{N}(D)$ is one for small $D$ and falls to zero when $D$ increases. An increase in $N$ also leads to a shift of the curves to the right, indicating a larger probability of jamming before $N$ grains flow freely. For disk, there have been a number of studies (both experimental $[1,8]$ and numerical [9-11]). The values of jamming probability we find for disks are sensibly larger than those reported in most previous studies. However, these previous works have followed a protocol where avalanches are triggered after a clog by perturbing the blocking arch. In a few cases, the system is emptied and made to flow again into the container keeping the aperture open [8]. In our case the silo is emptied and 
refilled with the aperture closed, which is opened only after the grains are static. Uñac et al. [12] have conducted a similar type of protocol as the one used here, but the system is tapped before opening the aperture to achieve a desired initial packing fraction. They found that clogging maw vary dramatically depending on the initial packing fraction before starting the avalanche.

Figure 3(a) shows a clear non-linear response when the number of vertexes of the grains are increased. From the most prone to jamming, the grain shapes can be ordered as: squares, hexagons, pentagons, triangles, disks and heptagons. It is unexpected that heptagons present a lower jamming probability than disks. However, this is observed only for small $D$. For values of $D$ where $J_{15}(D)$ is small, heptagons have a larger jamming probability. If one is interested in clogging developing after a larger number of grains have passed, the peculiar behavior of heptagons is partially lost. This can be seen in Fig. 3(b). For $J_{50}(D)$, for example, heptagons present a larger tendency to jamming than disk for most values of $D$. It is important to bear in mind that, when the orifice is opened, a few grains initially supported by the base fall through right away, affecting the statistics of jamming for small $N$. However, if one focuses on large avalanches, a steady flow develops before an arch is formed. Hence, results for large $\mathrm{N}$ are less affected by the statistics of the flow of the initial group of grains.

A non-linear behavior on the number of vertexes of the grains has also been found in the packing fraction of tapped columns of regular polygons [7] and tapped packings of aggregates [? ]. However, the packing fraction is significantly determined by the ability of a polygonal shape to tessellate the space, and one can naturally expect that tessellating shapes will yield higher packing fractions. In the case of clogging, the ability of interlocking thanks to the vertexes seems more significant. It is worth mentioning that hexagons and pentagons display very close values for $J_{N}(D)$ which make difficult to decide which clogs more. If their order is swapped in the list, the only singular shape becomes the triangle, which is much less prone to clogging than squares, pentagons and hexagons. We have carried out some preliminary simulations using a different "unclogging" protocol (where arches are broken every time they form to trigger a new avalanche) and we have found that in this case triangles present the larger jamming probability. A detailed discussion on the protocol dependence of clogging will be presented elsewhere.

\section{Conclusions}

We have shown that grains having polygonal shapes present significant differences in their clogging probabil- ity when they flow through small apertures. In general, the lower the number of vertexes of the regular polygon grains the larger is their tendency to jamming. However, triangles display a peculiar behavior, showing lower jamming probabilities than squares, pentagons and hexagons.

We have observed that the avalanche size distributions present exponential tails as it has been observed for circular and spherical grains in the past.

It is important to bear in mind that we have chosen to compare grains of different shapes keeping the mass (area) constant. However, other criteria may be used such as keeping the same diameter or perimeter for all shapes. As we have shown for the flow rate through large apertures this choice alters the results to a large extent [6].

\section{Acknowledgements}

This work has been supported by ANPCyT (Argentina) through grant PICT 2012-2155 and UTN (Argentina) through grants PID MAUTNLP0002184 and PID IFI1871.

\section{References}

[1] A. Janda, I. Zuriguel, A. Garcimartín, L. A. Pugnaloni and D. Maza, Eur. Phys. Lett. 84, 44002 (2008).

[2] C. C. Thomas and D. J. Durian, Phys. Rev. Lett. 114, 178001 (2015).

[3] I. Zuriguel, A. Garcimartín, D. Maza, L. A. Pugnaloni and J. M. Pastor, Phys. Rev. E 71, 051303 (2005).

[4] Box2D Physics Engine, www.box2d.org

[5] E Catto, Iterative dynamics with temporal coherence, available at

http://box2d.googlecode.com/files/GDC2005 ErinCatto.zip (retrived on October 2010).

[6] E. Goldberg, C. M. Carlevaro and L. A. Pugnaloni, Papers in Physics 7, 070016 (2015).

[7] C. M. Carlevaro and L. A. Pugnaloni, J. Stat. Mech. P01007 (2011).

[8] K. To, Phys. Rev. E 71, 060301 (2005).

[9] C. F. M. Magalhães, J. G. Moreira and A. P. F. Atman, Phys. Rev. E 82, 051303 (2010).

[10] G. Pérez, Pramana 70, 989 (2008).

[11] R. Arévalo, I. Zuriguel, Soft Matter 12, 123 (2016).

[12] R. O. Uñac, A. M. Vidales and L. A. Pugnaloni, J. Stat. Mech. P04008 (2012).

[13] L. K. Roth and H. M. Jaeger, Soft Matter 12, 1107 (2016). 